\title{
The effects of ventilation tubes versus no ventilation tubes for recurrent acute otitis media or chronic otitis media with effusion in 9 to 36 month old Greenlandic children, the SIUTIT trial: study protocol for a randomized controlled trial
}

\author{
Malene Nøhr Demant ${ }^{*}$, Ramon Gordon Jensen $^{1}$, Janus Christian Jakobsen ${ }^{2,3}$, Christian Gluud ${ }^{2}$ \\ and Preben Homøe $e^{1,4^{*}}$
}

\begin{abstract}
Background: The prevalence of otitis media in Greenlandic children is one of the highest in the world. International studies have shown that otitis-prone children may benefit from tubulation of the tympanic membrane. However, it is unknown whether these results can be applied to Greenlandic children and trials on the effects of ventilation tubes in high-risk populations have, to our knowledge, never been conducted.

Methods: The trial is an investigator-initiated, multicentre, randomized, blinded superiority trial of bilateral ventilation tube insertion versus treatment as usual (no tube) in Greenlandic children aged 9-36 months with chronic otitis media with effusion or recurrent acute otitis media. With randomization stratified by otitis media subtype and trial site, a type 1 error of 5\% and a power of $80 \%$, a total of 230 participants are needed to detect a decrease of two visits to a health clinic during 2 years, which is considered the minimal clinical relevant difference. The primary outcome measure will be assessed blindly by investigating medical records. Secondary outcome measures are number of episodes of acute otitis media, quality of life, number of episodes of antibiotics administration and proportion of children with tympanic membrane perforations.

Discussion: This trial will provide evidence-based knowledge of the effects of ventilation tubes in children with middle ear infections from the high-risk Greenlandic population. Furthermore, this trial will improve the understanding of conducting randomized clinical trials in remote areas, where management of logistical aspects is particularly challenging.
\end{abstract}

Trial registration: ClinicalTrials.gov, NCT02490332. Registered on 14 February 2016.

Keywords: Otitis media, Randomized clinical trials, Ventilation tubes, Grommets

\footnotetext{
*Correspondence: malenedemant@gmail.com; prho@regionsjaelland.dk ${ }^{1}$ Department of Otorhinolaryngology and Maxillofacial Surgery, Zealand University Hospital, Køge, Denmark

Full list of author information is available at the end of the article
} 


\section{Background}

Otitis media is one of the most common reasons for children to contact health clinics and insertion of ventilation tubes in the tympanic membrane remains the most frequent type of paediatric surgery in the USA as well as the main reason for prescription of out-ofhospital antibiotics to paediatric patients [1-3]. The worldwide socioeconomic consequences of the disease are substantial, owing to treatment and management of the disease and parental absence from work [4].

The prevalence of otitis media and other acute respiratory tract infections in Greenlandic children is one of the highest in the world; $20 \%$ of schoolchildren have impaired hearing in the frequencies of normal speech [5-10]. This pattern is also seen in, for example, the indigenous population in Australia [11]. Many theories have been suggested to explain the high prevalence in certain indigenous populations, such as anatomical features, poverty or limited access to health-care, as well as a high bacterial load in the nasopharynx [12-15]. Studies have shown that the prevalence of chronic suppurative otitis media in the two largest towns in Greenland, Nuuk and Sisimiut, is 9\% to 14\%, which is a public health problem requiring urgent attention, according to the World Health Organization $[6,7,12]$. A total of $91 \%$ of children with chronic suppurative otitis media develop permanent hearing loss, which underlines the importance of treatment and management of the disease [9].

Previous studies have shown that risk factors for the development of chronic suppurative otitis media are associated with the number of upper respiratory tract infections, as well as attendance of day care, mothers' educational status, passive smoking and socioeconomic factors, similar to known risk factors for acute otitis media found in other studies worldwide [7, 8, 10, 16]. It has been suggested that children with chronic otitis media with effusion, as well as recurrent acute otitis media, are more prone to develop chronic suppurative otitis media, and treatment of these conditions might therefore decrease the number of children with chronic suppurative otitis media $[6,17]$. The cumulative incidence of chronic suppurative otitis media in Greenland is $14 \%$ at the age of 4 , with the highest hazard rate between 6 and 12 months [7]. This indicates that the disease develops early in childhood, and calls for intervention as early as possible in otitis-prone children, to limit progression to chronic perforations.

Ventilation tubes are inserted in the tympanic membrane to equalize pressure and allow drainage of middle ear fluid.

According to guidelines from the UK, the USA and Denmark [1, 3, 18-21], there are two indications for the insertion of ventilation tubes in children:

- Chronic otitis media with effusion and hearing loss
- Recurrent acute otitis media

Chronic otitis media with effusion is defined as fluid in the middle ear cavity lasting $\geq 3$ months. Recurrent acute otitis media is defined as $\geq 3$ episodes of acute otitis media within 6 months or $\geq 4$ episodes of acute otitis media within 12 months [21].

Guidelines on the treatment of otitis media with effusion are fairly similar and have been recently updated in both the USA and the UK $[3,20]$. However, guidelines on treatment of recurrent acute otitis media vary and different guidelines currently exist in the USA, while there are no national guidelines in the UK $[1,19,21]$. This might explain part of the observed international differences in the number of ventilation tube insertions for recurrent acute otitis media [22].

The mentioned international guidelines on treatment with ventilation tubes are generally based on evidence of low methodological quality concerning such outcomes as number of otitis media episodes after treatment, quality of life after treatment, reduction in chronic tympanic membrane perforations after treatment and number of episodes of aural discharge after treatment, according to the GRADE evaluation of quality of evidence [18, 21, 23, 24]. Furthermore, it is primarily children from Western trial populations, and not children from high-risk otitis-prone populations, such as the Inuit in Greenland, who have been randomized in the previous trials. Currently, there are no national guidelines or programmes to ensure prevention and treatment of otitis media and impaired hearing in Greenland. The Greenlandic Ministry of Health has considered introducing ventilation tube insertion as a more consistent treatment modality in order to decrease the burden of otitis media in the country. However, trials on the effects of ventilation tubes among children in highrisk populations have, to our knowledge, never been conducted. We therefore argue that it is both medically and ethically necessary to conduct a randomized clinical trial before ventilation tubes are made part of the routine treatment of children with chronic otitis media with effusion and recurrent acute otitis media in Greenland. Moreover, ventilation tube treatment is currently not a part of standard care in Greenland and this provides a unique opportunity to investigate the unbiased effect of the treatment, which would not be possible in populations where ventilation tube treatment is already standard treatment.

\section{Methods/Design \\ Objective and hypothesis}

The primary objective of the trial will be to assess the effects of bilateral insertion of ventilation tubes versus 'treatment as usual' with no ventilation tubes in children 9-36 months old with chronic otitis media with effusion or recurrent acute otitis media in Greenland, measured by 
number of visits to health clinic for 2 years after randomization, assessed by investigating medical records.

The null hypothesis is:

- There is no difference in the number of visits to health clinics for children with chronic otitis media with effusion or recurrent otitis media treated with bilateral ventilation tubes, compared with children not treated with ventilation tubes.

\section{Design}

We have designed an investigator-initiated, parallelgroup, multicentre, randomized clinical superiority trial of bilateral ventilation tube insertion versus treatment as usual (no ventilation tube) in Greenlandic children with chronic otitis media with effusion or with recurrent acute otitis media.

The Consolidated Standards of Reporting Trials (CONSORT) flow chart for the trial is shown in Fig. 1 [25]. The Standard Protocol Items: Recommendations for Interventional Trials (SPIRIT) participant timeline is given in Table 1 and the SPIRIT checklist is given in Additional file 1 [26].

We will consider all patients followed at a participating trial site for participation and include patients if they comply with the inclusion and exclusion criteria listed in Table 2.

The inclusion procedure is shown in Fig. 2.

We will include children aged 9-36 months. Infants younger than this age group require special anaesthesiological care, which cannot be offered in Greenland for ventilation tube insertion. The upper age limit has been set as low as possible in order to intervene before progression of the disease, while ensuring adequate sample size in this small population. We have attempted to reduce the number of exclusion criteria to as few as possible. However, children with orofacial cleft, Down's syndrome and immune deficiency are, for other reasons, known to be at very high risk of chronic otitis media with effusion and recurrent acute otitis media and are therefore not comparable with other children. Children already treated with ventilation tubes can also not be included.

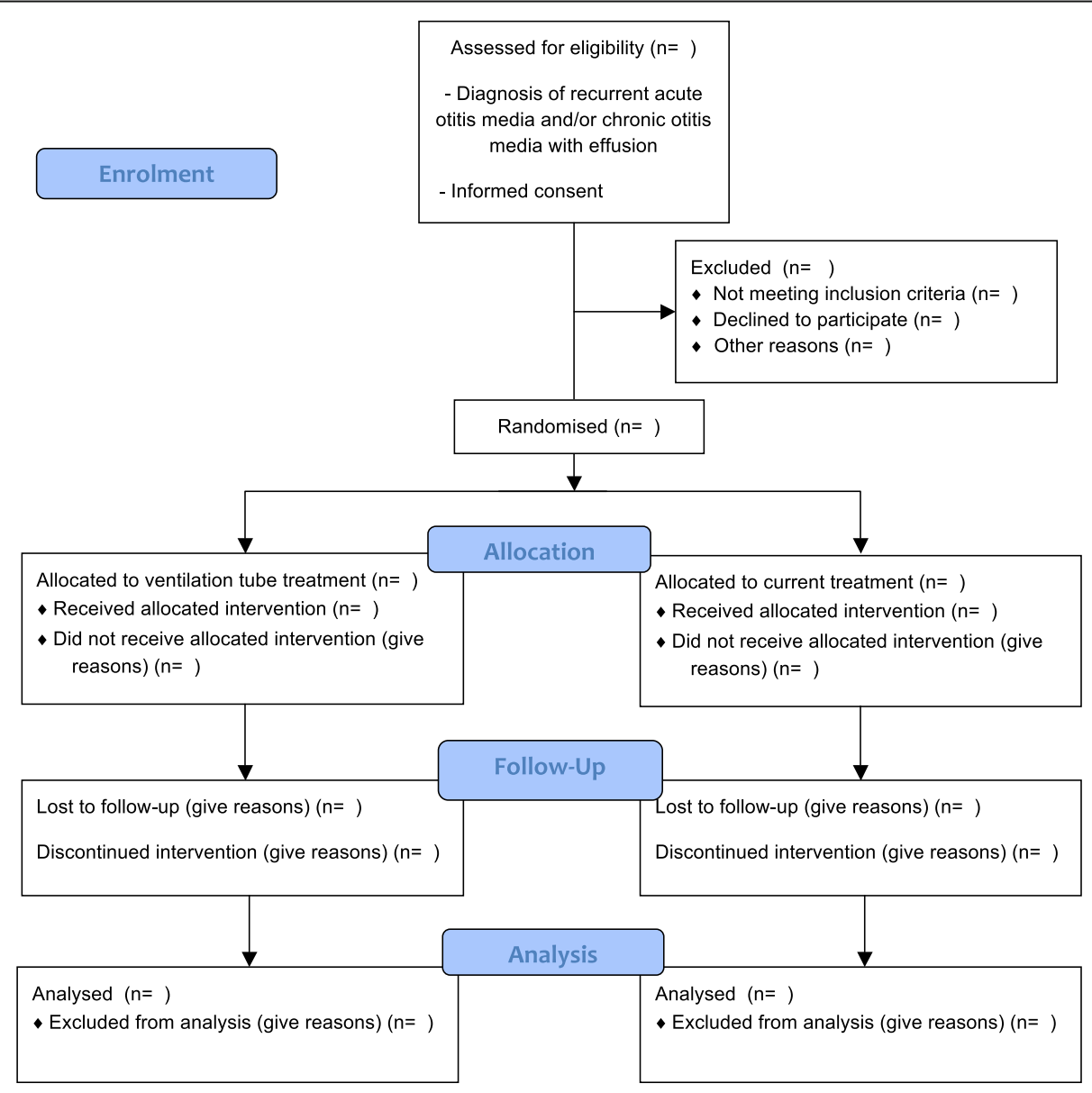

Fig. 1 Consolidated Standards of Reporting Trials (CONSORT) trial flow chart 
Table 1 Participant timeline, Standard Protocol Items: Recommendations for Interventional Trials (SPIRIT) diagram

\begin{tabular}{|c|c|c|c|c|c|c|}
\hline \multirow[b]{2}{*}{ Time point } & \multicolumn{6}{|c|}{ Study period } \\
\hline & Enrolment & Allocation & & & & Close-out \\
\hline Enrolment: & $-t_{1}$ & 0 & $t_{1}$ & $t_{1}+3$ months & $t_{1}+1$ year & $t_{1}+2$ years \\
\hline Eligibility screen & $x$ & & & & & \\
\hline Informed consent & $x$ & & & & & \\
\hline Allocation & $x$ & & & & & \\
\hline Interventions: & & $x$ & & & & \\
\hline Ventilation tube insertion & & & $x$ & & $x$ & \\
\hline No ventilation tube insertion & & & $x$ & & $x$ & \\
\hline \multicolumn{7}{|l|}{ Assessments: } \\
\hline Sex, age, ethnicity, socioeconomic factors & $x$ & & & & & \\
\hline Number of visits to health clinic & & & & & & $x$ \\
\hline Questionnaires: Otitis Media-6 and Caregiver impact & & $x$ & $x$ & $x$ & $x$ & $x$ \\
\hline Safety variables & & & & & & $x$ \\
\hline
\end{tabular}

\section{Trial site and personnel}

The trial sites are hospitals and health clinics in six Greenlandic towns: Nuuk, Sisimiut, Ilulissat, Aasiaat, Qaqortoq and Tasiilaq. The Greenlandic health-care system is divided into primary and secondary sectors. The only large and secondary referral hospital is in the capital, Nuuk [27].

The personnel performing the initial selection and screening of participants will be the regular staff at the Greenlandic health clinics and hospitals, for instance nurses, assistants and doctors of different specialities and ranks.

\section{Randomization}

The enrolment of patients will be conducted by an ear, nose and throat (ENT) specialist and the coordinating investigator. We will use centralized stratified web-based randomization. Prior to randomization, a computer will generate randomization sequences with varying block sizes that are unknown to the investigators. An internetbased randomization system will be set up conducting randomization stratified according to centre (trial site) and type of otitis media, i.e., recurrent acute otitis media or chronic otitis media with effusion at baseline (yes or no). The randomizing investigator will access the internet site through a personal information number. Patients who meet both criteria for chronic otitis media with effusion and recurrent acute otitis media will be considered in the recurrent acute otitis media group. The patients will be randomly allocated 1:1 into the two intervention groups.

\section{Interventions}

The experimental intervention consists of bilateral insertion of ventilation tubes (Donaldson) in the tympanic membranes, administered under general anaesthesia.

Short-term ventilation tubes will be used, consistent with the tube type applied in the majority of other studies, and in accordance with the type that is expected to be introduced in Greenland [18, 23]. If the tympanic

Table 2 Inclusion and exclusion criteria

\begin{tabular}{|c|c|}
\hline Inclusion criteria & Exclusion criteria \\
\hline Children aged 9-36 months & Children with orofacial cleft \\
\hline Children with at least one Greenlandic born parent and at least one Greenlandic born grandparent & Children with Down's syndrome \\
\hline American Society of Anaesthesiologists' physical status classification class 1 and 2 & $\begin{array}{l}\text { Children with known generalized immune } \\
\text { deficiency }\end{array}$ \\
\hline $\begin{array}{l}\text { B-type curve, defined as flat line tympanograms or gradient }<0.04 \mathrm{ml} \text {, or C2-type curve, defined } \\
\text { as pressure } \leq-200 \mathrm{dPa} \text {, measured by tympanometry at two visits three-four months apart } \\
\text { or }\end{array}$ & Children formerly treated with ventilation tubes \\
\hline $\begin{array}{l}\text { three episodes of acute otitis media in } 6 \text { months according to medical charts } \\
\text { or }\end{array}$ & $\begin{array}{l}\text { Lack of signed informed consent, signed by the } \\
\text { legal guardian }\end{array}$ \\
\hline tour episodes of acute otitis media in 12 months according to medical charts & \\
\hline Signed informed consent, signed by the legal guardian & \\
\hline
\end{tabular}




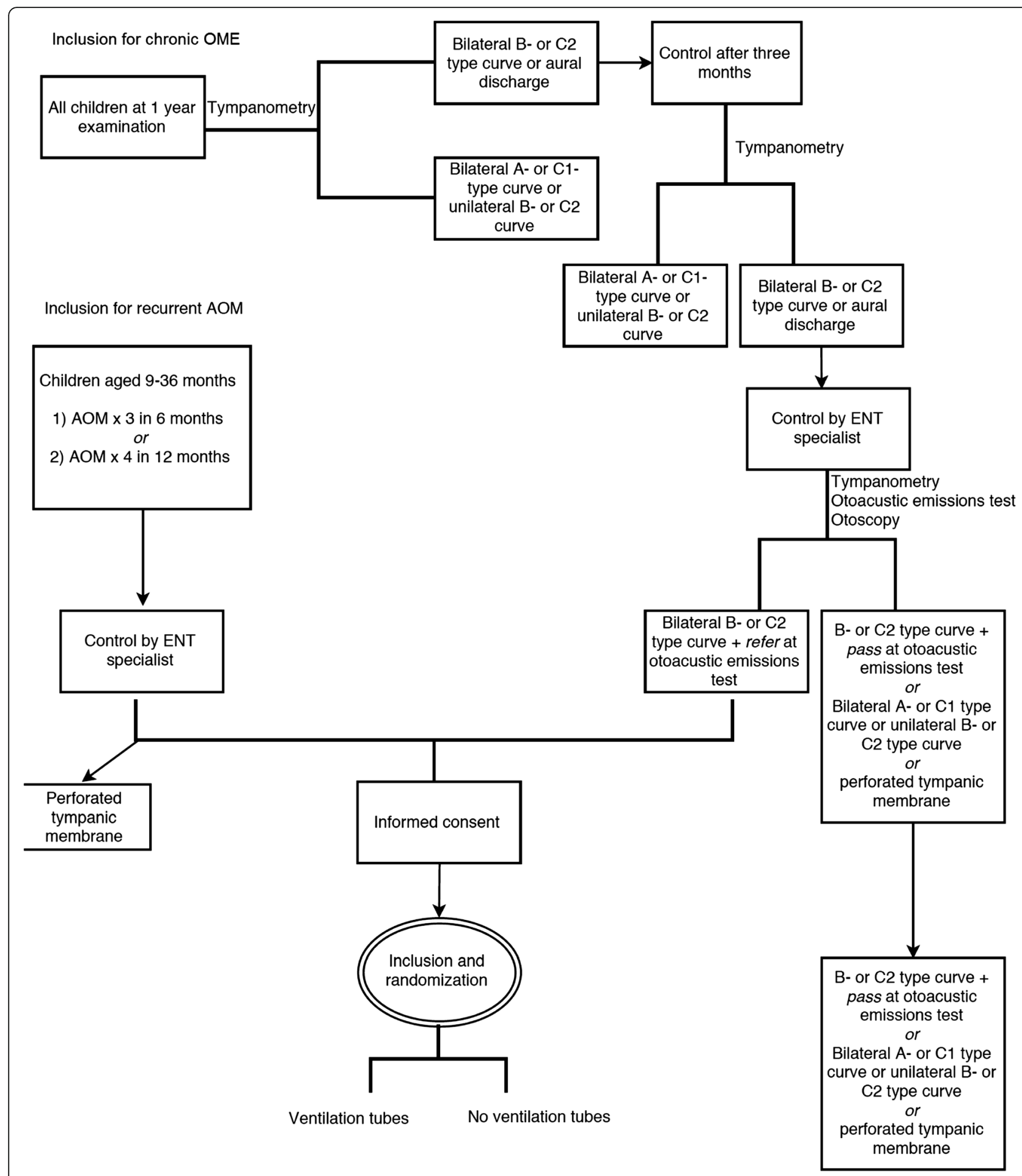

Fig. 2 Inclusion procedure flowchart. AOM, acute otitis media; ENT, ear, nose and throat; OME, otitis media with effusion

membrane is infected at the time of ventilation tube insertion, topical antibiotics will be given (Cilodex ${ }^{\odot}$ [dexamethasone + ciprofloxacin] eardrop suspension, $3 \mathrm{mg}+$ $1 \mathrm{mg}$, dexamethasone + ciprofloxacin) at a dose of four drops twice daily for 5 days.
If children in the intervention group seek medical assistance for ear problems after the insertion of ventilation tubes, these ear problems will be treated according to current practice in Greenland, which includes systemic antibiotic treatment (amoxicillin 40-90 $\mathrm{mg} /(\mathrm{kg}$ day)), as 
well as aural toilette and topical antibiotics (ciprofloxacin $1 \mathrm{ml} / 3 \mathrm{mg}$, three drops, twice daily, or Cilodex ${ }^{\circ}$ [dexamethasone + ciprofloxacin] eardrop suspension, $3 \mathrm{mg}+$ $1 \mathrm{mg}$, four drops twice daily for $5-7$ days).

The control intervention will be based on the current practice in Greenland, which includes systemic antibiotic treatment (amoxicillin, 40-90 $\mathrm{mg}(\mathrm{kg}$ day)), as well as aural toilette and topical antibiotics (ciprofloxacin $1 \mathrm{ml} /$ $3 \mathrm{mg}$, three drops, twice daily). Children in the control group will not be offered ventilation tubes for any circumstance until at least 2 years after the first ENT visit and randomization.

Children in both the intervention group and control group will have an ENT examination, including otoscopy by an ENT specialist at least once a year and at the end of the study period, 2 years after randomization.

\section{Outcomes}

To assess the primary outcome in as unbiased a manner as possible, it is necessary to choose an outcome that does not require visualization of the tympanic membrane, as this might reveal the trial intervention allocation of the patient (the ventilation tube or sequelae to such a tube would be visible). Therefore, the primary outcome will be the number of visits to health clinics during 2 years after randomization, determined according to the medical records, assessed blinded to intervention. In otherwise healthy children, the number of visits to health clinics can be assumed to reflect the number of episodes related to the ear or upper respiratory tract, as this is thought to be the primary reason for contact to health clinics for children aged 9-36 months [28].

It is currently not possible to assess hearing level as an outcome measure because there are no facilities available in Greenland able to meet the high standard of hearing evaluation, which would be necessary to detect differences in hearing levels of $4 \mathrm{~dB}$ [23]. We therefore postulate that an effect of ventilation tubes on otitis media with effusion might be reflected in a change in the number of visits to health clinics. The number of episodes of acute otitis media, based on medical records, will be included as a secondary outcome measure but will not be used as a primary outcome measure because the treatment providers and the outcome assessors will often not be sufficiently blinded to the trial intervention allocation.

\section{Primary outcome measure}

This is the number of visits to health clinic during 2 years after the randomization, based on medical records, evaluated by designated assessors blinded to the intervention.

\section{Secondary outcome measures}

1. Number of episodes of acute otitis media during the 2 years after the randomization, based on medical records, evaluated by designated assessors blinded to the intervention

2. Quality of life, measured on a $0-100$ scale by the validated Otitis Media-6 questionnaire [29, 30] and the Caregiver Impact Questionnaire [31, 32], assessed at randomization, 3 months after randomization, 1 year after randomization and at the end of the trial, 2 years after randomization

3. Number of episodes during the 2 years after randomization where oral or intravenous antibiotics have been administered, based on medical records, evaluated by designated assessors blinded to the intervention

4. Proportion of children with unilateral or bilateral tympanic membrane perforations in the intervention and control group at the end of the trial 2 years after randomization, based on otoscopical photos, which will be anonymized and evaluated by an ENT specialist without knowledge of the intervention

5. Serious adverse events during the 2 years after the randomization: any adverse event that results in death, is life threatening, requires hospitalization or prolongation of existing hospitalization or results in persistent or significant disability or incapacity [33]

\section{Exploratory outcomes}

1. Number of episodes of aural discharge during the 2 years after randomization, based on medical records, evaluated by designated assessors blinded to the intervention

The primary outcome measure as well as secondary outcome measures 1 and 3 and the exploratory outcome measure will be based on medical records, while secondary outcome measure 4 will be based on clinical examination. Secondary outcome measure 2 will be based on questionnaires; the first questionnaires will be completed at the clinical examination related to randomization, the remaining questionnaires will be sent to the participants' legal guardians by email and completed online.

\section{Blinding}

Owing to the type of the intervention, blinding of patients, parents and caregivers is not possible. However, the outcome assessors will be blinded to intervention and we also consider the number of visits to health clinics according to medical records (the primary outcome measure) as blinded. Outcome assessors will be ENT specialists. 
Blinding of the number of visits at the health-care centre is ensured by initial blinding of medical records by an investigator, and hereafter evaluation by two outcome assessors calculating the number of visits from the medical records. A third assessor may provide further evaluation in the event of any disagreement. Blinding of otoscopical results is ensured by the use of otoscopical photos, which will be anonymized and evaluated by an ENT specialist without knowledge of the intervention.

Blinding of quality-of-life measures cannot be obtained as the child and the parents are not blinded for the intervention. Therefore, this outcome measure must be considered at high risk of bias.

\section{Assessment of adverse events}

Adverse events and adverse reactions will be assessed at every ENT visit.

\section{Participant discontinuation and withdrawal}

Parents of participating children can withdraw their consent to participate at any time. To be able to analyze data at an intention-to-treat basis the investigator must ask for permission to use already collected data for data analysis.

The investigator or treating physician may discontinue the patient from further participation in the trial if the patient is diagnosed with any of the exclusion criteria. The investigator and treating physician will encourage the patient to continue the follow-up assessment and previously collected data should be used in further analysis.

We will monitor adherence to the control regimen. Parents of participating children will be reminded, at the time point for intervention and assessments, by email. Those who do not adhere to the control regimen specified will be further contacted by phone call and email.

\section{Data management}

Data will be entered in the data management system Easy Trial. Easy Trial hold standards according to the Danish Data Protection Agency, i.e., data are stored on private servers. Case report forms in electronic format will be used, as well as case report forms on paper. The paper case report forms will be entered in the data management system twice by two personnel independently to promote data quality. The personnel will otherwise not be related to the trial. Patients will be identified by patient identification number, which is also used at randomization. The trial is conducted according to regulations by the Danish Data Protection Agency and only people related to the trial and the central randomization centre will have access to data.

Missing data will be minimized by checking the completed questionnaires when returned to the investigators. If there are any missing answers, the parents of the included children will emailed a request to supply the missing answers.

\section{Statistical plan and data analysis}

Based on power $(1-\beta)=0.80, \alpha=0.05$ (two-sided) and standard deviation of five visits to health clinics and

- An estimated eight visits to health clinics during 2 years in the control group and

- An estimated six visits to health clinics during 2 years in the experimental group

we need a sample size of 99 individuals in each intervention group.

As we do not expect that data are normally distributed, the non-parametric van Elteren test will be used; thus, we obtain $99 / 0.86=115$ participants per intervention group or 230 participants in total [34].

The two interventions will be compared regarding all outcomes. The analysis of the outcomes will be based on the intention-to-treat principle, i.e., all randomized participants will be included in the analysis, regardless of how much treatment they have received. Per-protocol analysis may be considered if important deviations from the protocol compromise the validity of the intentionto-treat analysis.

Dichotomous outcomes will be analyzed using logistic regression, continuous outcomes will be analyzed using linear regression and count data will be analyzed using the van Elteren test [34]. Our primary analysis will be adjusted for the stratification variables used in the randomization (trial site and type of otitis media). In secondary analysis, we will adjust all analyses (except when non-parametric tests are used) for additional significant design variables (age, sex, attending daycare, smokers in the household, diet, family history of otitis media). The statistical analysis will be described in detail in a separate paper published before the analysis of the trial results begins.

If only data are missing on the dependent outcome, we will use per-protocol data but we will interpret out results with caution if these missing data potentially bias our results. Otherwise, if more than $5 \%$ of the outcome data are missing, multiple imputation will be used (STATA 14). However, the 5\% cut-off is not definitive. The imputation result will be considered the primary overall result. This analysis will be supplemented by the following sensitivity analyses:

1. 'Best-worst-case' scenario: It will be assumed that all participants lost to follow-up in the experimental group have a mean score +2 standard deviations and have no event; and all those with missing outcomes in the control group have a mean score of -2 standard deviations and have an event.

2. 'Worst-best-case' scenario: It will be assumed that all participants lost to follow-up in the experimental 
group have a mean score of -2 standard deviations and have an event; and all those lost to follow-up in the control group have a mean score +2 standard deviations and have no event [35].

Results from both scenarios will be presented in our trial publication.

If the null hypotheses on the primary outcome measures are not rejected, our main conclusion will be that we found no significant difference between the two interventions. The analysis of the remaining outcome measures will be presented for hypothesis-generating purposes.

\section{Discussion}

This trial will provide evidence-based knowledge of the effects of ventilation tubes in children with middle ear infections. Furthermore, the effects of ventilation tube administration in a high-risk population, such as the Greenlandic, have never, to our knowledge, been investigated and this trial will improve the understanding of conducting randomized clinical trials in remote areas, where management of logistical aspects is particularly challenging.

The strengths of this trial are the inclusion of children at high risk of developing otitis media and sequelae thereof; the central randomization regarding both generation of allocation sequence and allocation concealment; the primary outcome measure that monitors use of the health-care system; and our attempts to blind as many outcome measures as possible. Our trial also has limitations. First, no updated systematic review of the effects of ventilation tubes is currently available. We refer to previous Cochrane reviews published in 2008 and 2010, respectively $[18,23]$ but the methodology of these reviews is not optimal and the literature search has not been sufficiently updated. We are writing a protocol for a systematic review assessing the effects of ventilation tubes; this protocol will be registered on PROSPERO. As soon the protocol is registered, we will perform a literature search and begin writing the review. Nevertheless, it is a major methodological limitation that we cannot sufficiently take into account a complete and valid overview of previous studies on the effects of ventilation tubes. Further methodological limitations are a lack of blinding to the intervention regarding a number of the outcome measures and potential problems with drop-out during follow-up, owing to lack of interest, migration or logistics.

\section{Dissemination policy}

The Greenlandic population will be informed of the trial as well as its final results through national media. All participating health clinics and hospitals will be visited by the coordinating investigator and instructed in objectives and screening or inclusion procedures. The final and interim results will be presented at NUNA MED, an international conference on Greenlandic medicine and health held every third year.

Trial results will be published in English, Danish and Greenlandic.

The Government of Greenland will be informed of the final results before a press release is issued but will have no influence on the reporting of the results.

\section{Trial status}

We launched the randomization on 18 February 2016. At the end of June, six children had been enrolled and randomly allocated to a group.

\section{Additional file}

Additional file 1: SPIRIT 2013 Checklist. (DOCX $61 \mathrm{~kb})$

\section{Abbreviation}

CONSORT: Consolidated Standards of Reporting Trials; ENT: ear, nose and throat; SPIRIT: Standard Protocol Items: Recommendations for Interventional Trials

\section{Acknowledgements}

The Greenland Institute of Natural Resources is thanked for unconditional economical support (grant no. 80.11).

\section{Funding}

This trial is funded by a grant from Greenland Institute of Natural Resources, grant no. 80.11. Other funding possibilities will be explored.

\section{Availability of data and materials}

The final dataset will be publically available in depersonalized format after the end of the trial on the Danish Data Archive and Zenodo.

\section{Authors' contributions}

MND participated in the study design, statistical calculations and study methodology and drafted the manuscript. RGJ contributed to the study design and methodology and helped to draft the manuscript. JCJ participated in study design and methodology, carried out statistical calculations and drafted the manuscript. CG participated in study design and methodology and helped draft the manuscript. PH designed the study and methodology and contributed to the manuscript. All authors read and approved the final manuscript. All trial results whether positive, negative or neutral, will be published, preferably in a peer-reviewed medical journal. All authorship will be determined according to International Committee for Medical Journal Editors guidelines for authorship [36]. The first author is coordinating investigator Malene Nøhr Demant and last author is responsible investigator Professor Preben Homøe.

\section{Competing interests}

The authors declare that they have no competing interests.

\section{Consent for publication}

Not applicable.

\section{Ethics approval and consent to participate}

The trial will be conducted in compliance with the protocol approved by the Greenlandic ethics committee (No. 2015-112556) and according to the International Committee on Harmonization - Good Clinical Practice standards, as well as the Greenlandic standards of Code of Conduct $[37,38]$. No deviation from the protocol will be implemented without the prior review and approval of the regulatory authorities, except where it may be necessary to eliminate an immediate hazard to the trial participants. In such cases, the deviation will be reported to the regulatory authorities as soon as possible. 
The personnel at the hospitals and health clinics will give written information about the trial to the child's legal guardian when the patient is first considered for screening or inclusion. Further oral and written information will be given at the first visit by the ENT specialist, where the written informed consent will be obtained.

It will be stressed that this is a research project, a randomized clinical trial, and that participation can be withdrawn at any time, without consequences for future treatment at the health clinic. The cultural aspects of respect of authorities as well as different ways of expressing decline will be considered. Furthermore, the trial is registered at ClinicalTrials.gov (NCT02490332) and approved by the Danish Data Protection Agency (no. 2015-41-4047). This trial is conducted according to regulations by the Danish Data Protection Agency. Only people related to the trial and the central randomization centre will have access to data.

\section{Author details}

'Department of Otorhinolaryngology and Maxillofacial Surgery, Zealand University Hospital, Køge, Denmark. ${ }^{2}$ Copenhagen Trial Unit, Centre for Clinical Intervention Research, Rigshospitalet, Copenhagen University Hospital, Copenhagen, Denmark. ${ }^{3}$ Department of Cardiology, Holbæk Hospital, Holbæk, Denmark. Institute of Clinical Medicine, Faculty of Health and Medical Sciences, University of Copenhagen, Copenhagen, Denmark.

Received: 5 July 2016 Accepted: 21 December 2016 Published online: 19 January 2017

\section{References}

1. Rosenfeld RM, Schwartz SR, Pynnonen MA, Tunkel DE, Hussey HM, Fichera JS, Grimes AM, Hackell JM, Harrison MF, Haskell H, Haynes DS, Kim TW Lafreniere DC, LeBlanc K, Mackey WL, et al. Clinical practice guideline: tympanostomy tubes in children. Otolaryngol Head Neck Surg. 2013; 149(1 Suppl):S1-35.

2. Sidell D, Shapiro NL, Bhattacharyya N. Demographic influences on antibiotic prescribing for pediatric acute otitis media. Otolaryngol Head Neck Surg. 2012;146(4):653-8

3. Rosenfeld RM, Shin JJ, Schwartz SR, Coggins R, Gagnon L, Hackell JM, Hoelting D, Hunter LL, Kummer AW, Payne SC, Poe DS, Veling M, Vila PM, Walsh SA, Corrigan MD. Clinical practice guideline: otitis media with effusion executive summary (update). Otolaryngol Head Neck Surg. 2016; 154(2):201-14.

4. Ahmed S, Shapiro NL, Bhattacharyya N. Incremental health care utilization and costs for acute otitis media in children. Lanyngoscope. 2014;124(1):301-5.

5. Homøe P, Jensen RG. Mellemørebetændelse i Grønland (Middle ear infections in Greenland). Månedsskrift for Almen Praksis (Journal of The Danish College of General Practitioners). 2013;91:228-37.

6. Homøe P, Christensen RB, Bretlau P. Prevalence of otitis media in a survey of 591 unselected Greenlandic children. Int J Pediatr Otorhinolaryngol. 1996:36(3):215-30

7. Koch A, Homøe P, Pipper C, Hjuler T, Melbye M. Chronic suppurative otitis media in a birth cohort of children in Greenland: population-based study of incidence and risk factors. Pediatr Infect Dis J. 2011;30(1):25-9.

8. Jensen RG, Homøe P, Andersson M, Koch A. Long-term follow-up of chronic suppurative otitis media in a high-risk children cohort. Int J Pediatr Otorhinolaryngol. 2011;75(7):948-54.

9. Jensen RG, Koch A, Homøe P. The risk of hearing loss in a population with a high prevalence of chronic suppurative otitis media. Int J Pediatr Otorhinolaryngol. 2013;77(9):1530-5.

10. Homøe P, Christensen RB, Bretlau P. Acute otitis media and sociomedical risk factors among unselected children in Greenland. Int J Pediatr Otorhinolaryngol. 1999:49(1):37-52.

11. Morris PS, Leach AJ, Silberberg P, Mellon G, Wilson C, Hamilton E, Beissbarth J. Otitis media in young Aboriginal children from remote communities in Northern and Central Australia: a cross-sectional survey. BMC Pediatr. 2005;5:27

12. WHO. Report of a WHO/CIBA foundation workshop 1996, prevention of hearing impairment from chronic otitis media, London, UK, 19-21 November 1996. Geneva: WHO; 1996.

13. Homøe P, Prag J, Farholt S, Henrichsen J, Hornsleth A, Kilian M, Jensen JS. High rate of nasopharyngeal carriage of potential pathogens among children in Greenland: results of a clinical survey of middle-ear disease. Clin Infect Dis. 1996;23(5):1081-90.
14. Jacoby P, Carville KS, Hall G, Riley TV, Bowman J, Leach AJ, Lehmann D. Crowding and other strong predictors of upper respiratory tract carriage of otitis media-related bacteria in Australian Aboriginal and non-Aboriginal children. Pediatr Infect Dis J. 2011;30(6):480-5.

15. Smith-Vaughan $H$, Byun $R$, Nadkarni M, Jacques NA, Hunter N, Halpin S, Morris PS, Leach AJ. Measuring nasal bacterial load and its association with otitis media. BMC Ear Nose Throat Disord. 2006;6:10.

16. Hoffman HJ, Daly KA, Bainbridge KE, Casselbrant ML, Homøe P, Kvestad E, Kvaerner KJ, Vernacchio L. Panel 1: Epidemiology, natural history, and risk factors. Otolaryngol Head Neck Surg. 2013;148(4 Suppl):E1-E25.

17. Tos M. Upon the relationship between secretory otitis in childhood and chronic otitis and its sequelae in adults. J Laryngol Otol. 2007;95(10):1011-22.

18. McDonald S, Langton Hewer CD, Nunez DA. Grommets (ventilation tubes) for recurrent acute otitis media in children. Cochrane Database Syst Rev. 2008:4:CD004741.

19. Lieberthal AS, Carroll AE, Chonmaitree T, Ganiats TG, Hoberman A, Jackson MA, Joffe MD, Miller DT, Rosenfeld RM, Sevilla XD, Schwartz RH, Thomas PA Tunkel DE. The diagnosis and management of acute otitis media. Pediatrics. 2013:131(3):e964-99.

20. National Institute for Health and Care Excellence. www.nice.org.uk/ guidance/CG60 [Accessed 2 January 2016]

21. Heidemann CH, Lous J, Berg J, Christensen JJ, Håkonsen SJ, Jakobsen M, Johansen CJ, Nielsen LH, Hansen MP, Poulsen A, Schousboe LP, Skrubeltang $C$, Vind $A B$, Homøe P. Danish guidelines on management of otitis media in preschool children. Int J Pediatr Otorhinolaryngol. 2016;87:154-63.

22. Schilder AGM, Lok W, Rovers MM. International perspectives on management of acute otitis media: a qualitative review. Int J Pediatr Otorhinolaryngol. 2004;68(1):29-36.

23. Browning GG, Rovers MM, Williamson I, Lous J, Burton MJ. Grommets (ventilation tubes) for hearing loss associated with otitis media with effusion in children. Cochrane Database Syst Rev. 2010;10:CD001801.

24. Guyatt GH, Oxman AD, Vist GE, Kunz R, Falck-Ytter Y, Alonso-Coello P, Schünemann HJ. GRADE: an emerging consensus on rating quality of evidence and strength of recommendations. BMJ. 2008;336(7650):924-6.

25. Moher D, Schulz K, Altman D. The CONSORT statement: revised recommendations for improving the quality of reports of parallel group randomized trials. BMC Med Res Methodol. 2001;1:2

26. Chan A-W, Tetzlaff JM, Altman DG, Laupacis A, Gøtzsche PC, Krleža-Jerić K, et al. SPIRIT 2013 statement: defining standard protocol items for clinical trials. Ann Intern Med. 2013;158:200-7.

27. Niclasen B, Mulvad G. Health care and health care delivery in Greenland. Int J Circumpolar Health. 2010;69(5):437.

28. Koch A, Sørensen P, Homøe P, Mølbak K, Pedersen FK, Mortensen T, Elberling $\mathrm{H}$, Eriksen AM, Olsen OR, Melbye M. Population-based study of acute respiratory infections in children, Greenland. Emerg Infect Dis. 2002;8(6):586-93.

29. Rosenfeld RM, Goldsmith AJ, Tetlus L. Quality of life for children with otitis media. Arch Otolaryngol Head Neck Surg. 1997;123(10):1049-54.

30. Heidemann $\mathrm{CH}$, Godballe C, Kjeldsen AD, Johansen EC, Faber CE, Lauridsen $\mathrm{HH}$. The Otitis Media-6 questionnaire: psychometric properties with emphasis on factor structure and interpretability. Health Qual Life Outcomes. 2013;11:201.

31. Boruk M, Lee $P$, Faynzilbert $Y$, Rosenfeld RM. Caregiver well-being and child quality of life. Otolaryngol Head Neck Surg. 2007;136:159-68.

32. Heidemann $\mathrm{CH}$, Godballe C, Kjeldsen AD, Johansen EC, Faber CE, Lauridsen $\mathrm{HH}$. Otitis media and caregiver quality of life: psychometric properties of the modified Danish Version of the Caregiver Impact Questionnaire. Otolaryngol Head Neck Surg. 2014;151(1):142-9.

33. European Parliament and the Council of the European Union. Directive 2001/20/EC of the European Parliament and of the Council of 4 April 2001 on the approximation of the laws, regulations and administrative provisions of the Member States relating to the implementation of good clinical practice in the conduct of clinical trials on medicinal products for human use. 2001. https://ec.europa.eu/health/files/eudralex/vol-1/dir_2001_20/dir 2001_20_en.pdf

34. Jakobsen JC, Tamborrino M, Winkel P. Haase N, Perner A, Wetterslev J, et al. Count data analysis in randomised clinical trials. J Biometrics Biostatistics. 2015;6:227.

35. Jakobsen JC, Wetterslev J, Winkel P, Lange T, Gluud C. Thresholds for statistical and clinical significance in systematic reviews with meta-analytic methods. BMC Med Res Methodol. 2014;14:120. 
36. International Committee of Medical Journal Editors, Recommendations, Defining the Role of Authors and Contributors, http://www.icmje.org/ recommendations/browse/roles-and-responsibilities/defining-the-role-ofauthors-and-contributors.html [Accessed 01 Jan 2016].

37. ICH harmonised tripartite guideline: guideline for good clinical practice. Essential documents for the conduct of a clinical trial. J Postgraduate Med. 2001;47(4):264-7.

38. Greenlandic Medical Society, Code of conduct. http://gmsnet.dk/test/wpcontent/uploads/2015/10/CodeOfConduct_final.pdf [Accessed 2 Jan 2016]

Submit your next manuscript to BioMed Central and we will help you at every step:

- We accept pre-submission inquiries

- Our selector tool helps you to find the most relevant journal

- We provide round the clock customer support

- Convenient online submission

- Thorough peer review

- Inclusion in PubMed and all major indexing services

- Maximum visibility for your research

Submit your manuscript at www.biomedcentral.com/submit
Biomed Central 